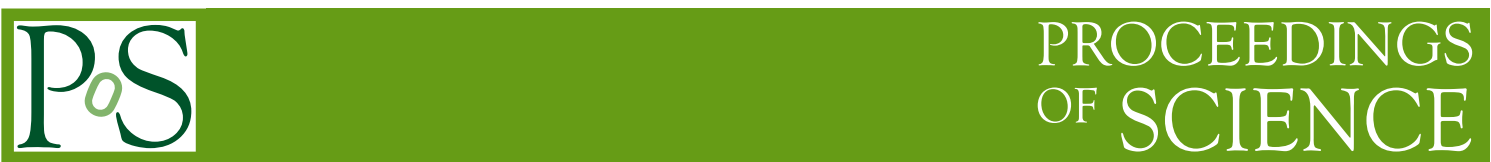

Neutrino Oscillations Results from the T2K Experiment

\author{
Laura Munteanu ${ }^{a, *}$ \\ ${ }^{a}$ IRFU, CEA Saclay, Gif-sur-Yvette, France \\ E-mail: laura.munteanu@cea.fr
}

The T2K (Tokai-to-Kamioka) experiment probes the mass differences and mixing of neutrinos through measurements of neutrino oscillations. A beam of muon neutrinos or muon antineutrinos is generated at the J-PARC proton accelerator on the east coast of Japan, and the beam's composition after oscillations is measured $295 \mathrm{~km}$ away in the Super-Kamiokande detector. The transition of muon neutrinos and antineutrinos to other flavors is governed by neutrino mixing and mass parameters, including the phase $\delta_{C P}$, which determines the amount of $\mathrm{CP}$ violation in neutrino mixing. Previous measurements from $\mathrm{T} 2 \mathrm{~K}$ have shown a strong constraint on $\delta_{C P}$ with the exclusion of a significant fraction of $\delta_{C P}$ values at $3 \sigma$ confidence. Here, we present the latest results from T2K with data collected through 2020.

40th International Conference on High Energy physics - ICHEP2020

July 28 - August 6, 2020

Prague, Czech Republic (virtual meeting)

\footnotetext{
${ }^{*}$ Speaker
} 
The relationship between the three neutrino mass eigenstates $\left(m_{1}, m_{2}, m_{3}\right)$ and the three flavor eigenstates $\left(v_{e}, v_{\mu}, v_{\tau}\right)$ is described by the Pontecorvo-Maki-Nakagawa-Sakata (PMNS) mixing matrix [1]. The latter is parametrized by three mixing angles $\left(\theta_{12}, \theta_{13}, \theta_{23}\right)$ and one complex charge-parity symmetry $(\mathrm{CP})$ violating phase, $\delta_{C P}$.

Neutrino oscillation parameters are extracted by measuring the event rates of different neutrino flavors far from the neutrino source. The probability of oscillation from one flavor to another depends on the distance a neutrino has travelled $(L)$, referred to as the experiment's "baseline", the neutrino energy $\left(E_{v}\right)$, the two mass differences between the mass eigenstates $\left(\Delta m_{i j}^{2}=m_{i}^{2}-m_{j}^{2}\right)$, and on the mixing angles, as well as possible matter effects.

Currently, constraints exist for the $\theta_{12}$ and $\theta_{13}$ angles, and for the two mass differences $\Delta m_{21}^{2}$ and $\Delta m_{32}^{2}$. The $\theta_{23}$ angle has a large uncertainty and it is not yet known whether it has a value of $\pi / 4$ (corresponding to maximal mixing), or above (below) this value (upper (lower) octant)). The other outstanding oscillation parameter is the complex phase $\delta_{C P}$. Any value of $\delta_{C P}$ different from 0 or $\pi$ would lead to $\mathrm{CP}$ violation in the leptonic sector, with $\pm \pi / 2$ representing maximal CP violation. One remaining task is the determination of the neutrino mass hierarchy $(\mathrm{MH})$, which can either be normal $(\mathrm{NH})$ or inverted $(\mathrm{IH})$. In addition to these measurements, the consistency of the PMNS framework is also probed.

Accelerator neutrino experiments produce a neutrino beam dominated by a specific flavor with a well known energy spectrum, measured close to the production point with a near detector facility. The beam is directed towards a far detector, where its composition is measured after oscillations and compared to simulations of theoretical predictions. Two types of phenomena are detected: initial flavor disappearance and final flavor appearance. The depth of the disappearance dip depends mainly on the value of the $\theta_{23}$ angle, whereas the position of its minimum depends on $\Delta m_{32}^{2}$. The appearance probability depends on the value of $\delta_{C P}$, the $\mathrm{MH}$, and the $\theta_{13}$ mixing angle. All of the PMNS parameters are measured simultaneously, so the above factorization is just for illustrative purposes.

\section{The T2K Experiment}

The T2K experiment is a long-baseline accelerator neutrino oscillation experiment, located in Japan [2]. A $v_{\mu}\left(\bar{v}_{\mu}\right)$ enhanced beam is produced at the Japan Proton Accelerator Research Complex (J-PARC) on the east coast of Japan. The neutrino beam is produced by directing $30 \mathrm{GeV}$ protons on to a graphite target, producing hadrons such as pions and kaons. These charged hadrons are then focused with a set of magnetic horns with polarity set to preferentially select positively (negatively) charged hadrons, which then decay producing mainly $v_{\mu}\left(\bar{v}_{\mu}\right)$. In order to measure the oscillation parameters, the beam composition is measured at two points: before oscillation, at the near detector complex at J-PARC; the composition after oscillations is detected with the Super-Kamiokande (SK) far detector, located in the Kamioka mine.

T2K uses the off-axis technique. The $v_{\mu}$ beam produced at J-PARC is offset by an angle of $2.5^{\circ}$ with respect to the SK detector direction. This focuses the neutrino energy distribution around 600 $\mathrm{MeV}$, maximizing oscillation probabilities, while also reducing the contamination of the $v_{\mu}$ beam by $v_{e}$ also produced in the hadron decays. 
The near detector complex consists of several subdetectors with specific physics goals. The INGRID detector is a scintillating detector located on-axis with respect to the beam and monitors the beam position and stability. The Near Detector at $280 \mathrm{~m}$ (ND280) consists of an upstream $\pi^{0}$ detector and two scintillating fine grained detector targets (FGD1 and FGD2), sandwiched between three time projection chambers (TPC). FGD1 is a hydrocarbon-based scintillating detector, whereas FGD2 contains water layers in addition to the hydrocarbon ones to generate interactions on oxygen. The ensemble is surrounded by an electromagnetic calorimeter and placed inside the former UA1 magnet. ND280 is therefore a magnetized detector, capable of separation of particle tracks based on their electric charge, enabling it to characterize the wrong-sign background $\left(\bar{v}_{\mu}\right.$ in the $v_{\mu}$-mode beam and vice-versa). ND280 is placed at the same off-axis angle as SK and provides constraints for the oscillation analysis by measuring the unoscillated spectrum. It is also used to perform neutrino cross-section measurements (along with the INGRID detector).

SK is a water-based Cherenkov detector. It consists of a cylindrical tank filled with 50 kton of pure water and has a fiducial volume of $22.5 \mathrm{kton}$. The inside of the detector is lined with 11,100 20" photo-multiplier tubes (PMTs) in order to detect the Cerenkov light emitted inside the detector, while the outside is lined with 1,8858 " PMTs to veto signals from cosmic ray particles. It reconstructs the flavor (electron or muon-like) of neutrinos based on Cherenkov ring patterns.

The T2K experiment has been taking data since 2009 in both $v_{\mu}$ and $\bar{v}_{\mu}$ mode and has accumulated a total of $1.97 \times 10^{21}$ protons-on-target (POT) in $v_{\mu}$ mode and $1.63 \times 10^{21}$ POT in $\bar{v}_{\mu}$ mode. In 2020, the beam facility has reached a stable operating beam power of $515 \mathrm{~kW}$.

\section{The T2K Oscillation Analysis Strategy}

T2K is sensitive to the oscillation parameters $\sin ^{2} \theta_{23}, \sin ^{2} \theta_{13}, \Delta m_{32}^{2}$ and $\delta_{C P}$, though its sensitivity to $\sin ^{2} \theta_{13}$ is much smaller than that of reactor experiments. It is also sensitive to a lower extent to the MH. A fit of the predicted event rate to the oscillated data at SK is performed, and the data at ND280 is used to provide precise predictions for the far detector spectrum. The latter is parametrized as a function of so-called "nuisance parameters" - all non-oscillation parameters which affect the T2K measurements at both the near and far detector, and thus parametrize the systematic uncertainties in the evaluation of the oscillation parameters. Two statistical frameworks are used: a frequentist sequential fit, in which the near detector data is fit and propagated as a set of constraints on nuisance parameters to the far detector; and a Bayesian simultaneous fit of the near and far detector data. In both cases, three sets of nuisance parameters are fit in order to constrain the oscillation parameters. The nuisance parameter sets and their inputs are:

- Flux uncertainties - the neutrino flux is simulated with the FLUKA simulation package, and then tuned with external data constraints from the NA61/SHINE hadron production experiment at CERN, to account for mis-modelled hadron interactions in the graphite target. For this analysis, T2K's flux tune was extracted from NA61/SHINE data obtained with a full-size T2K replica target [3], whereas in previous analyses the tune used data obtained with a thin graphite target. This reduced the systematic uncertainties on the flux from $8 \%$ to $5 \%$ before the near detector fit.

- Detector systematics - the detector systematic uncertainties are parametrized according to each detector (ND280 or SK). 
- Neutrino cross-section uncertainties - the neutrino interaction model is tuned to world crosssection data and our understanding of neutrino-nucleus interaction theory.

At the energy scale of $\mathrm{T} 2 \mathrm{~K}$, the dominant interaction type is the charged-current quasi-elastic reaction (CCQE), in which a neutrino interacts with a nucleon and produces a charged lepton and a nucleon in the final state. This type of reaction also has the advantage of describing a two-particle final state interaction and allows the neutrino energy to be reconstructed using only information from the outgoing lepton. Other charged-current interactions are possible, the most prevalent of which are resonant pion production (CCRES), multi-nucleon so-called $2 \mathrm{p} 2 \mathrm{~h}$ interactions, and deep inelastic scatters (CCDIS).

Data and simulated samples at ND280 are classified according to their final state pion multiplicity, beam mode, and which target the interaction occurred in. The three sample categories are: the charged-current pion-less topology (CC0 $\pi$, enriched in $\mathrm{CCQE}$ ), single pion topology $(\mathrm{CC} 1 \pi$, enriched in CCRES) and multi-pion topology (CCOther, enriched in CCDIS). This sample classification is applied separately to events originating in the FGD1 target (carbon nuclei) and the FGD2 target (carbon and oxygen nuclei), as well as for different muon charge selections. T2K also uses selections to control the $v_{\mu}$ contamination when running in $\bar{v}_{\mu}$ mode ("wrong-sign" samples).

The cross-section uncertainties are designed to cater to the dominant reaction types and the features of each detector. The main differences in the neutrino cross-section model with respect to the one used in previous analyses are:

- A tuned Benhar Spectral Function to describe CCQE interactions [4], with a smaller uncertainty on the nucleon removal energy compared to previous analyses [5];

- An added energy dependent freedom to the Nieves et al. model [6] describing multi-nucleon (2p2h) interactions;

- Correlated pion final state interaction (FSI) uncertainties between the near and the far detector and an improved treatment of multi-pion and deep inelastic scattering events.

Combining the flux, detector and cross-section uncertainty models described above, the data obtained with the near detector is fit in order to extract constraints on the nuisance parameters and their correlations. The p-value to obtain the observed data given the model before the near detector fit is $74 \%$. The near detector constraint reduces the systematic error on the number of observed events at SK from $\sim 11 \%$ to $\sim 4 \%$ across all single-ring samples at the far detector.

The SK data is categorized in a similar way to that of ND280. Samples are defined according to the Cherenkov ring multiplicity. Four single-ring (CCQE enriched) samples are defined to select $v_{e}\left(v_{\mu}\right)$ enriched events by requiring electron-like (muon-like) rings for both beam modes. An additional sample, enriched in pion-production events ( $\mathrm{CC} 1 \pi$ topology), is defined by events with one electron-like ring along with the signature of a Michel decay electron from a pion decay.

\section{Results}

The appearance results are summarized in a plot of the number of neutrino and antineutrino mode electron-like candidates, shown in Fig. 1. This plot compares the measured number of data events in the $v_{e}\left(\bar{v}_{e}\right)$ samples at SK with theoretical predictions that differ as a function of the values of $\sin ^{2} \theta_{23}, \delta_{C P}$ and the MH. T2K data appear to slightly favour $\mathrm{NH}$, the upper octant of $\sin ^{2} \theta_{23}$ and maximal $\mathrm{CP}$ violation. 


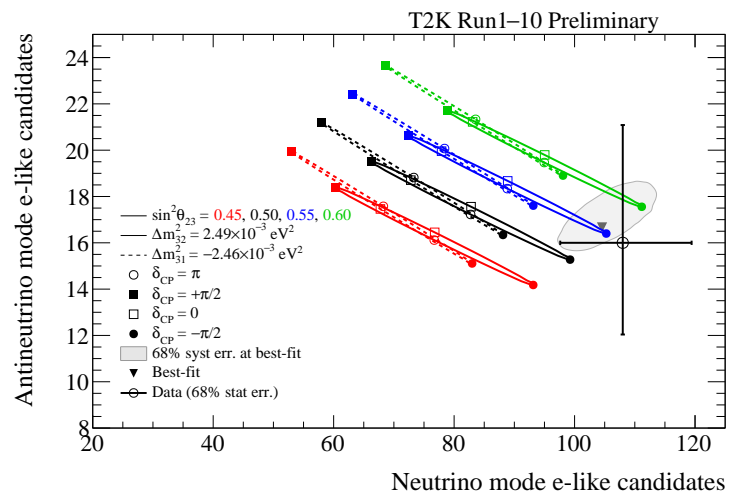

Figure 1: Plot of the number of neutrino and antineutrino mode electron-like candidates comparing different theoretical predictions (ellipses) to the $\mathrm{T} 2 \mathrm{~K}$ data and its statistical uncertainty (point with error bars), and to the data fit best-fit point and error (gray triangle and shaded gray area). Solid (dashed) ellipses represent $\mathrm{NH}$ (IH) predictions. The color of each ellipse corresponds to a different value of $\sin ^{2} \theta_{23}$. The points along the ellipses mark the predictions for different $\delta_{C P}$ values.

The best fit values and intervals on the oscillation parameters are extracted by fitting the oscillated $v_{\mu}\left(\bar{v}_{\mu}\right)$ and $v_{e}\left(\bar{v}_{e}\right)$ spectra at SK. This fit uses the constraints on the flux and crosssection systematic parameters obtained by fitting the unoscillated spectra at the near detector and marginalizing over the systematic parameters. The error contours for the oscillation parameters are presented in Fig. 2. Fig. 2a shows that the CP conserving values of 0 and $\pi$ are excluded at the $90 \%$

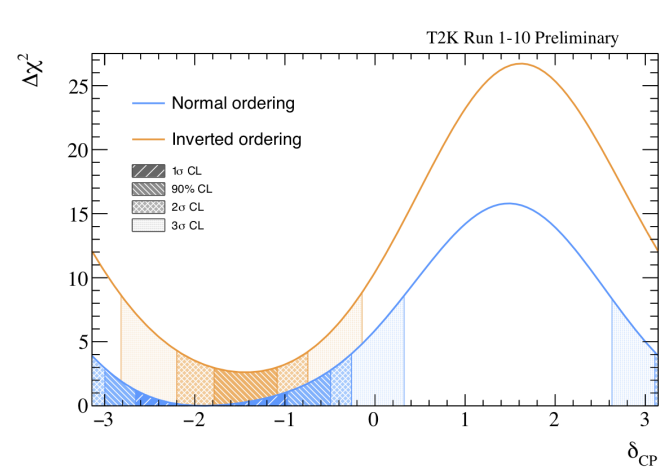

(a) $\Delta \chi^{2}$ profile as a function of $\delta_{C P}$

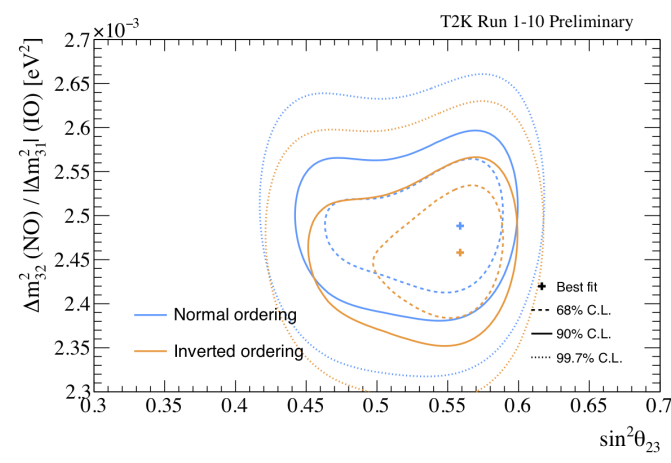

(b) $2 \mathrm{D}$ confidence level contours in $\Delta m_{32}^{2}\left(\Delta m_{31}^{2}\right.$ for IH) vs. $\sin ^{2} \theta_{23}$

Figure 2: Oscillation parameter results, including reactor constraint. (a) 1D $\Delta \chi^{2}$ distribution as a function of $\delta_{C P}$. The shaded confidence intervals were computed using the Feldman-Cousins method. (b) Data fit 2D confidence level contours in $\Delta m_{32}^{2}\left(\Delta m_{31}^{2}\right.$ for IH) vs. $\sin ^{2} \theta_{23}$. The contours for a given mass hierarchy are computed using the best fit $\chi^{2}$ in each hierarchy.

confidence level (C.L.) under the NH hypothesis. In addition, $35 \%$ of $\delta_{C P}$ values are excluded at the $3 \sigma$ level under the NH hypothesis, and the limits are stronger for IH. Fig. $2 \mathrm{~b}$ shows the contours in the $\sin ^{2} \theta_{23}-\Delta m_{32}^{2}$ space. The Bayesian analysis results showed that the data display an $80.8 \%$ preference for $\mathrm{NH}$ and a $77.1 \%$ preference for the upper octant.

T2K tests the fitting framework and systematic error model through simulated data studies. 
Alternative models or tunes are used to create mock data sets and passed through the oscillation analysis procedure. The results are compared to a maximum sensitivity fit in which the nominal simulation is fit to itself. These studies allow to identify biases and motivate model improvements. In case of a significant deviation this effect is included in the oscillation parameter errors.

After performing multiple such robustness studies, we saw that none of the simulated data sets introduce an effect big enough to change the conclusions regarding the $90 \%$ C.L. exclusion of $\delta_{C P}$ $\mathrm{CP}$ conserving values of 0 and $\pi$. The largest effect of any the robustness studies was used to extend the $\delta_{C P} 90 \%$ confidence interval [3.00, 0.49] by 0.073 units to the left (towards $-\pi$ ) and by 0.080 units to the right (i.e. towards $\pi$ ). The largest observed bias was on the $\Delta m_{32}^{2}$ parameter and was added as an additional uncertainty of $1.4 \times 10^{-5} \mathrm{eV}^{2} / \mathrm{c}^{4}$.

\section{Conclusion and Future Prospects}

Future prospects for T2K include performing two sets of joint fits, with the NOvA and SK collaborations respectively. These joint fits will allow degeneracies to be lifted in both experiments, notably regarding the neutrino mass hierarchy.

J-PARC also plans an upgrade of the neutrino beamline, aiming to reach $750 \mathrm{~kW}$ by 2022 [7], which will allow T2K to considerably increase its statistics. This motivates the need for a better control of systematic uncertainties. In order to respond to this need, T2K will install an upgrade of the ND280 detector in 2022 [8], whose design is based on better reconstruction, lower particle detection thresholds and larger acceptance. T2K will continue taking data in order to further improve the precision of neutrino oscillation measurements.

\section{References}

[1] Ziro Maki, Masami Nakagawa, and Shoichi Sakata. Remarks on the unified model of elementary particles. Prog. Theor. Phys., 28:870-880, 1962.

[2] K. Abe et al. The T2K Experiment. Nucl. Instrum. Meth. A, 659:106-135, 2011.

[3] N. Abgrall et al. Measurements of $\pi^{ \pm}, K^{ \pm}$and proton double differential yields from the surface of the T2K replica target for incoming $31 \mathrm{GeV} / \mathrm{c}$ protons with the NA61/SHINE spectrometer at the CERN SPS. Eur. Phys. J. C, 79(2):100, 2019.

[4] O. Benhar, A. Fabrocini, S. Fantoni, and I. Sick. Spectral function of finite nuclei and scattering of GeV electrons. Nucl. Phys. A, 579:493-517, 1994.

[5] K. Abe et al. Constraint on the matter-antimatter symmetry-violating phase in neutrino oscillations. Nature, 580(7803):339-344, 2020.

[6] J. Nieves, I. Ruiz Simo, and M.J. Vicente Vacas. The nucleon axial mass and the MiniBooNE Quasielastic Neutrino-Nucleus Scattering problem. Phys. Lett. B, 707:72-75, 2012.

[7] K. Abe et al. J-PARC Neutrino Beamline Upgrade Technical Design Report. 82019.

[8] K. Abe et al. T2K ND280 Upgrade - Technical Design Report. 12019. 